\title{
A nomogram to predict survival in patients with acute-on-chronic hepatitis B liver failure after liver transplantation
}

\author{
Liang Chen ${ }^{1,2 \#}$, Jiebin Zhang ${ }^{1,2 \#}$, Tongyu Lu ${ }^{1,2 \#}$, Jianye Cai ${ }^{1,2}$, Jun Zheng ${ }^{1,2}$, Jia Yao ${ }^{1,2}$, Shuhong Yi ${ }^{1,2}$, \\ Hua $\mathrm{Li}^{1,2}$, Guihua Chen ${ }^{1,2}$, Hui Zhao ${ }^{1,2}$, Yingcai Zhang ${ }^{1,2}$, Yang Yang ${ }^{1,2}$ \\ ${ }^{1}$ Department of Hepatic Surgery and Liver Transplantation Center, Organ Transplantation Institute, The Third Affiliated Hospital of Sun Yat-Sen \\ University, Guangzhou, China; ${ }^{2}$ Guangdong Key Laboratory of Liver Disease Research, The Third Affiliated Hospital of Sun Yat-Sen University, \\ Guangzhou, China \\ Contributions: (I) Conception and design: L Chen, J Zhang, T Lu; (II) Administrative support: Y Yang, G Chen; (III) Provision of study materials or \\ patients: S Yi, H Li, G Chen, Y Zhang; (IV) Collection and assembly of data: J Zhang, H Zhao, J Yao; (V) Data analysis and interpretation: L Chen, J \\ Zhang, T Lu; (VI) Manuscript writing: All authors; (VII) Final approval of manuscript: All authors. \\ \#These authors contributed equally to this work. \\ Correspondence to: Hui Zhao; Yingcai Zhang; Yang Yang. Department of Hepatic Surgery and Liver Transplantation Center, The Third Affiliated \\ Hospital of Sun Yat-Sen University, Guangzhou 510630, China. Email: zhaohui8@mail.sysu.edu.cn; zhangyc3@mail.sysu.edu.cn; yysysu@163.com.
}

Background: Individualized prediction of survival after liver transplantation (LT) for patients with hepatitis B virus-related acute-on-chronic liver failure (HBV-ACLF) has not been well investigated. This study aimed to develop a prognostic nomogram for patients with HBV-ACLF undergoing LT.

Methods: The nomogram was derived from a retrospective study of 290 patients who underwent LT for HBV-ACLF at the Third Affiliated Hospital of Sun Yat-sen University between January 2012 and December 2017. Concordance index and determiner calibration curve was used to ascertain the predictive accuracy and discriminative ability of the nomogram. The predictive performance of the nomogram was compared with that of Child-Pugh score, model for end-stage liver disease (MELD), MELD-Na, chronic liver failure Consortium Organ Failure score (CLIF-C OFs), and CLIF-C ACLF.

Results: The 1-year mortality rate was $23.1 \%$ (67/290). The Cox multivariate analysis showed that risk factors for 1-year survival rate included white blood cell count, alanine aminotransferase/aspartate aminotransferase ratio, and the organ failure numbers. The determiner calibration curve showed good agreement between prediction of the nomogram and actual observation. The concordance index of the nomogram for predicting 1-year survival was 0.707 , which was significantly higher than that of other prognostic models: Child-Pugh score (0.626), MELD (0.627), MELD-Na (0.583), CLIF-C OF (0.674), and comparable to that of CLIF-C ACLF (0.684).

Conclusions: Our study developed a novel nomogram that could accurately predict individualized posttransplantation survival in patients with HBV-ACLF. The nomogram might be a useful tool for identifying HBV-ACLF patients who would benefit from LT.

Keywords: Acute-on-chronic liver failure (ACLF); liver transplantation (LT); hepatitis B virus (HBV); nomogram

Submitted Aug 30, 2020. Accepted for publication Jan 03, 2021.

doi: 10.21037/atm-20-6180

View this article at: http://dx.doi.org/10.21037/atm-20-6180 


\section{Introduction}

Acute-on-chronic liver failure (ACLF) is a complex syndrome characterized by severe jaundice, encephalopathy, and coagulative disorders. The 4-week mortality rate among patients with ACLF ranges from 29.6-73.0\% depending on severity and grade (1-3). The clinical management of ACLF currently focuses on multiorgan-supportive care and treatment of complications.

For ACLF patients who do not recover spontaneously and experience worsening of the disease, liver transplantation (LT) is the only definite treatment with a good outcome. It has been reported that ACLF patients who receive LT have a 1-year survival rate of approximately $80 \%$ $(2,4,5)$. However, most studies published to date have been retrospective with a limited sample size. Despite the significant improvements in patient and graft survival over time, the clinical application of LT in ACLF is hampered by the concern that it may be futile for patients with multiple organ failure $(4,6)$. Thus, more practical algorithms are required to assess individual mortality risk (7).

Currently, the prognosis of patients with ACLF undergoing LT cannot be accurately predicted. Several scoring systems have been developed to predict posttransplant outcomes including the Survival Outcomes Following Liver Transplant score (SOFT) (8), Donor age $\times$ recipient Model for End-stage Liver Disease score (D-MELD) (9), and University of California Los Angeles score (UCLA) (6); however, the prognostic values of these models have not been sufficiently validated in the context of LT for hepatitis B virus (HBV)-related ACLF patients. In the prediction of waiting list mortality, conventional models such as the MELD score, MELD-Na score, and ChildPugh score (CPS) have shown unsatisfactory predictive ability due to their neglect of the systemic inflammatory status of ACLF patients (10). Thus, it remains difficult to evaluate the individual specific prognosis of HBV-ACLF patients who undergo LT, or to offer advice to severe patients on whether they are suitable for LT.

Nomograms are graphical depictions of complex predictive statistical models for individual patients (11). With user friendly digital interface and increased accuracy, the use of nomograms aids patients and physicians in clinical decision-making. Nomograms have also been proven to be reliable prognostic tools for predicting waiting list mortality in cirrhotic LT candidates (12), as well as survival in decompensated cirrhotic patients without ACLF (13). However, nomograms for HBV-ACLF patients undergoing
LT have rarely been reported. Therefore, this study aimed to develop a nomogram to investigate mortality risk in patients with HBV-ACLF undergoing LT based on preoperative baseline variables, and to compare its predictive ability with that of other prognostic models.

We present the following article in accordance with the Transparent Reporting of a multivariable prediction model for Individual Prognosis or Diagnosis (TRIPOD) Checklist (available at http://dx.doi.org/10.21037/atm-20-6180).

\section{Methods}

The study was conducted in accordance with the Declaration of Helsinki (as revised in 2013). The study was approved by institutional ethics committee of The Third Affiliated Hospital of Sun Yat-Sen University (No. 2018-353-02), and individual consent for this retrospective analysis was waived. The study outcomes will not affect the future management of the patients. All patient data were obtained from the medical record system of The Third Affiliated Hospital of Sun Yat-Sen University and have been secured.

\section{Study design and population}

Medical records were reviewed retrospectively to identify patients with HBV-ACLF who underwent LT at the Liver Transplantation Center of the Third Affiliated Hospital of Sun Yat-Sen University from January 2012 to December 2017. The diagnosis of ACLF corresponded to the 2009 consensus recommendations of the Asian Pacific Association for the Study of the Liver (APASL) (14). All adult patients who underwent LT for the treatment of HBV-ACLF were included in the study. The patients were divided into 2 subgroups according to the European Association for the Study of the Liver (EASL)-ACLF criteria (2). Pretransplant evaluation included chest/abdominal computed tomography (CT) scans, a cardiopulmonary evaluation, and cancer screening to assess the hepatic vasculature and exclude extrahepatic malignancies. Patients listed with non-HBV hepatitis, multiple organ transplantation, retransplantation, or cancer were excluded, as were those under the age of 18 years old. The outcomes of each patient were recorded as survival or death.

Before LT, all patients received standard-of-care treatment according to the APASL consensus recommendations (14). A similar triple immunosuppressive regimen including 
corticosteroids, tacrolimus, and mycophenolate mofetil was administered to all patients. Patients with pre-transplant renal failure received basiliximab as induction therapy. Prophylaxis for HBV reactivation following LT was based on a combination of hepatitis B immune globulin and antiviral therapy. No patients were lost to follow-up (15).

\section{Data collection}

Clinical characteristics, laboratory measurements [e.g., aspartate aminotransferase (AST), alanine aminotransferase (ALT), prothrombin activity (PTA), international normalized ratio (INR), total bilirubin (TB), albumin (ALB), and creatinine (Cr)], HBV-DNA levels, and other clinical information of recruited patients were collected from hospital electronic records within the week before transplantation. Organ failure (OF) was determined based on the chronic liver failure Consortium (CLIF-C) OF scoring system at the time of LT (16). Those with failure of 5 or 6 organs were combined into 1 group, as they constituted a relatively small sample size. The CPS, MELD, MELD-Na, CLIF-C OFs, and CLIF-C ACLF scores were calculated as previously described (16).

\section{Statistical analysis}

Data analyses were performed with SPSS version 23.0 for Windows (IBM Corp., Chicago, IL, USA) and R software version 3.4.4 (Institute for Statistics and Mathematics, Vienna, VIC, Austria). Survival analysis was performed using MedCalc version 17.9.7 (MedCalc Software, Ostend, Belgium). The optimal cut-off values for total points calculated with nomogram were determined using $\mathrm{X}$ tile software version 3.6.1 (Yale University, New Haven, CT, USA). Normally distributed variables were expressed as mean \pm standard deviation and compared using the Student's $t$-test; other quantitative variables were described as median [25-75\% interquartile range (IQR)] and compared using the Mann-Whitney $U$ test. Categorical variables were expressed as frequencies and percentages, and were compared using the $\chi^{2}$ test.

Univariate analysis and multivariate Cox proportional hazards models were performed to identify significant variables associated with 1-year survival. Hazard ratios (HR) and $95 \%$ confidence intervals $(\mathrm{CI})$ were calculated. A twotailed $\mathrm{P}$ value of $<0.05$ was considered to be statistically significant. Kaplan-Meier analysis was used to perform survival analysis among subgroups, and the differences were tested with a log-rank test.

The nomogram was established based on the results of multivariate Cox proportional hazards analysis using $\mathrm{R}$ software. The performance of the nomogram was evaluated by Harrell's concordance index (C-index) and determiner calibration plots. Comparisons between nomogrampredicted and observed Kaplan-Meier estimates of survival probability were performed, and 1000 bootstrap resamples were applied. Comparisons between $\mathrm{C}$-indices of the nomogram, CPS, MELD score, MELD-Na, CLIF-C OFs, and CLIF-C ACLF were performed using the R package 'survIDINRI' (17). Also, an online version of the nomogram was developed using the R package 'DynNom'.

\section{Results}

\section{Baseline characteristics of patients with $H B V-A C L F$ undergoing $L T$}

After the exclusion of patients who did not meet the inclusion criteria, 290 patients with HBV-ACLF who underwent LT were enrolled from 664 patients initially screened (Figure 1). The median follow-up time was 21.5 months (IQR, 8.0-42.3 months). The patients had a median pre-transplant MELD score of 35.0 (IQR, 29.041.0, Table 1). The cohort was predominantly male and had a high rate of ascites. Liver and coagulation failure were the most common types of organ failure among participants ( $74.8 \%$ and $60.7 \%$, respectively). Patients with failure of 3 or more organs accounted for $35.5 \%$ of the cohort. The 90 -day and 1 -year post-LT mortality was $21.0 \%$ and $23.1 \%$, respectively (Table S1). Most deaths occurred during the 28-day and 90-day periods following LT. On the basis of the EASL-ACLF criteria, 218 (75.2\%) patients were diagnosed with ACLF. "Non-ACLF" was used to refer to patients who had ACLF according to the APASL criteria but not according to the EASL-CLIF criteria. Most patients were classified as ACLF grade $2(100 / 218)$ or 3 (103/218). The organ failure distribution in the ACLF group was similar to that of the entire cohort, with liver and coagulation failure as the most common types of failure $(91.3 \%$ and $78.0 \%$, respectively). White blood cells (WBC), NLR, PTA, ALT, AST, TBIL, and creatinine were significantly worse in the ACLF group. Patients who met the EASL-ACLF criteria also had significantly higher MELD, MELD-Na, CPS, CLIF-C OFs, and CLIF-C ACLF scores than those who did not. The 90-day and 1-year mortality rates in the ACLF 


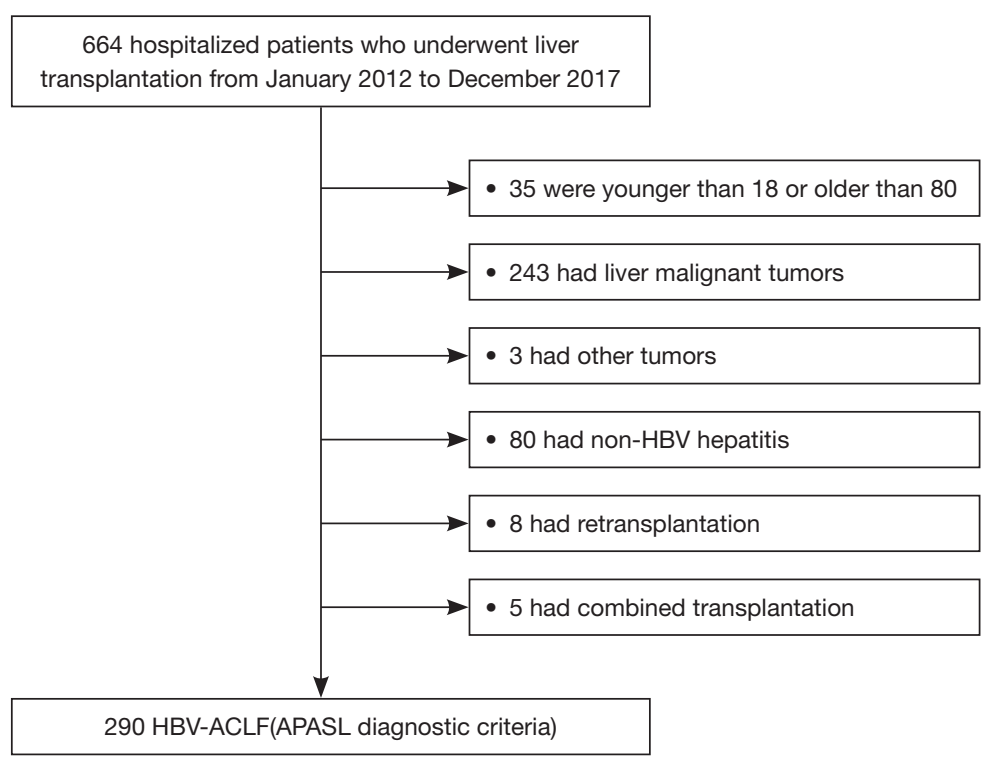

Figure 1 Flowchart of the study's inclusion and exclusion process. ACLF, acute-on-chronic liver failure; APASL, Asian Pacific Association for the Study of the Liver; HBV, hepatitis B virus.

Table 1 Baseline characteristics of HBV-ACLF patients undergoing liver transplantation

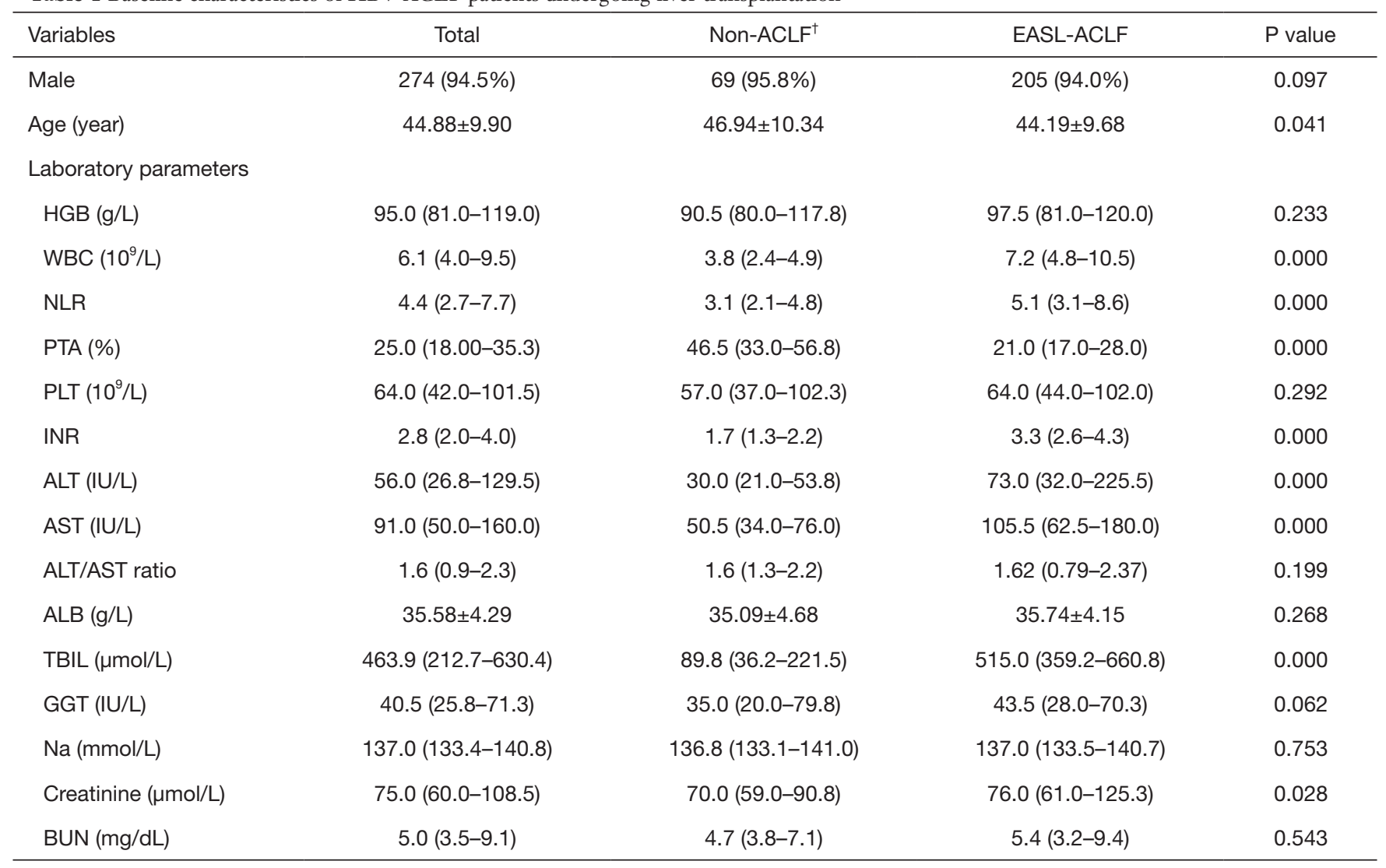

Table 1 (continued) 
Table 1 (continued)

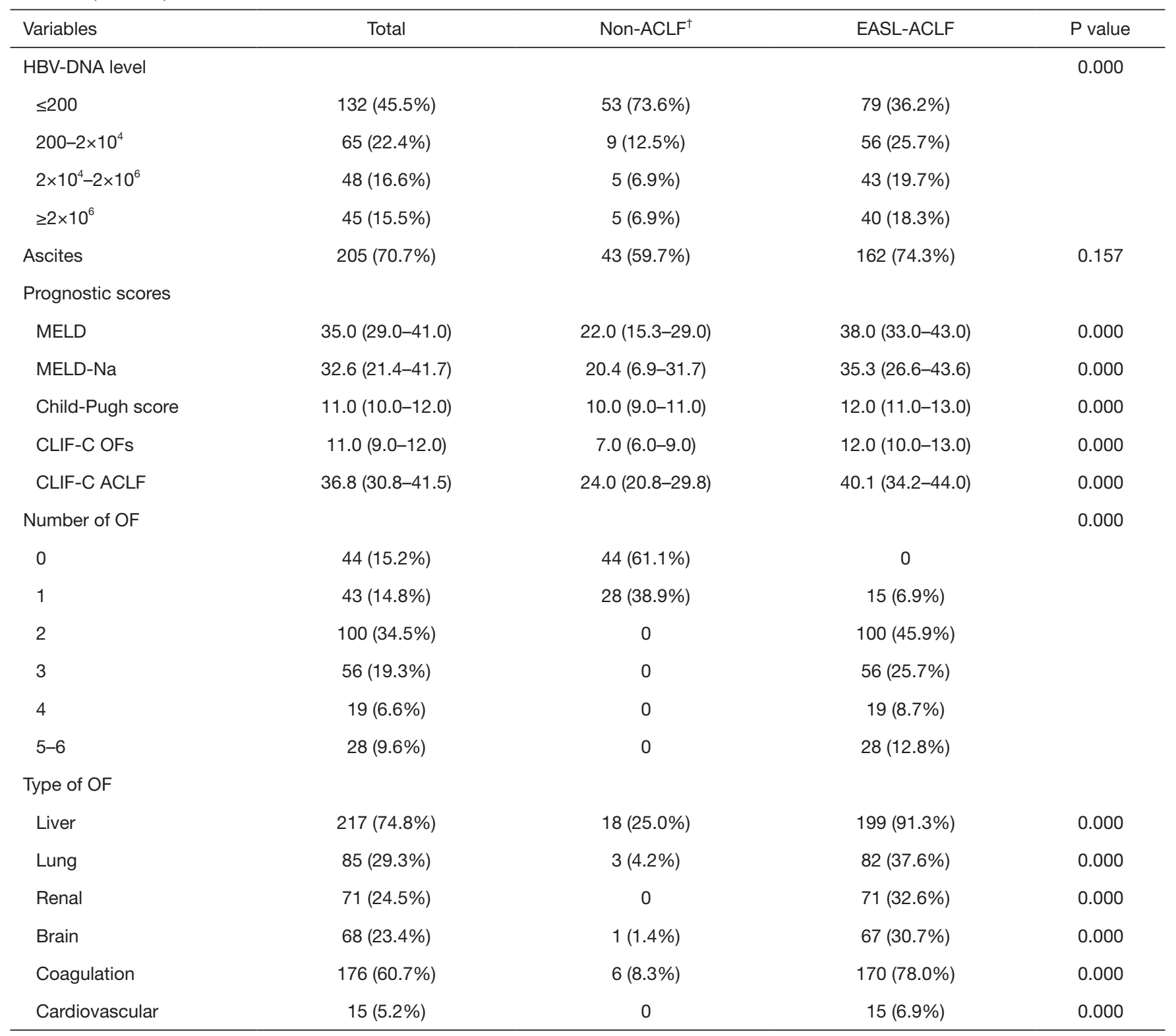

$\mathrm{P}$ values $<0.05$ represent statistical significance. Where applicable, data are presented as $\mathrm{n}(\%)$, median (interquartile range) or mean \pm standard deviation. ${ }^{\dagger}$, non-ACLF indicates patients satisfying the APASL ACLF criteria but not the EASL criteria. ALT/AST ratio, ALT to AST ratio; ACLF, acute-on-chronic liver failure; ALB, albumin; ALT, alanine transaminase; AST, Aspartate transaminase; BUN, blood urea nitrogen; CLIF-C OF, chronic liver failure Consortium Organ Failure score; EASL, European Association for the Study of the Liver; GGT, gamma-glutamyl transpeptidase; HBV, hepatitis B virus; HGB, hemoglobin; INR, international normalized ratio; MELD, model for end stage liver disease; NLR, neutrophil-to-lymphocyte ratio; OF, organ failure; PTA, prothrombin activity; PLT, platelet; TBIL, total bilirubin; WBC, white blood cell.

group were significantly higher than those in the nonACLF group (24.8\% vs. 9.7\%, $\mathrm{P}<0.05 ; 27.1 \%$ vs. $11.1 \%$, $\mathrm{P}<0.05$, respectively). Patients with ACLF grade 3 also had a poorer prognosis than those with ACLF grade 1 or 2 .

\section{Risk factors for predicting 1-year post-transplant mortality}

Baseline clinical and laboratory parameters for the prediction of 1-year post-LT mortality was investigated using a univariate Cox analysis regression model. The 
univariate analysis indicated that among the 20 studied factors, WBC (HR 1.05, P<0.001), NLR (HR 1.045, $\mathrm{P}=0.008$ ), ALT/AST ratio (HR 1.078, $\mathrm{P}=0.033$ ), creatinine (HR 1.002, $\mathrm{P}=0.001$ ), HBV-DNA (HR 1.318, $\mathrm{P}=0.008$ ), liver failure (HR 2.083, $\mathrm{P}=0.032$ ), lung failure (HR 3.237, $\mathrm{P}<0.001$ ), renal failure (HR 2.483, $\mathrm{P}<0.001$ ), brain failure (HR 3.134, $\mathrm{P}<0.001$ ), cardiovascular failure (HR 4.882, $\mathrm{P}<0.001$ ), and the organ failure numbers (HR 1.552, $\mathrm{P}<0.001)$ were significantly associated with 1 -year mortality (Table 2). Then, the 11 significant factors above were entered into the multivariate Cox proportional hazards model, which indicated that WBC (HR 1.050, 95\% CI: 1.002-1.101, $\mathrm{P}=0.041$ ), ALT/AST ratio (HR 1.095, 95\% CI: $1.031-1.163, \mathrm{P}=0.003$ ), and the organ failure numbers (HR 1.470, 95\% CI: $1.234-1.750, \mathrm{P}<0.001)$ were statistically associated with the 1 -year mortality of participants. Participants were stratified by the organ failure numbers, and their post-LT survival was examined (Table S2). An ascending trend in mortality risk was observed for patients with a higher number organs with failure; those with 5-6 failing organs had the highest 1 -year post-LT mortality rate (62.29\%), which was approximately $2-4$ times the mortality rate of other subgroups. Also, to analyze the role of $\mathrm{OF}$ in post-LT mortality, patients were divided by the type of OF (Figure S1, Table S3). Patients with cardiovascular (66.67\%), brain (42.65\%), and lung (41.18\%) failure had higher 1-year mortality rates than those with other types of $\mathrm{OF}$.

\section{Prognostic nomogram for 28-day, 3-month, 6-month, and 1-year mortality}

To predict the 28-day, 3-month, 6-month, and 1-year mortality of patients with HBV-ACLF who underwent LT, a prognostic nomogram was built based on the 3 significant independent factors derived from the multivariate Cox regression analysis (Figure 2). According to the point scale, each parameter within the nomogram was assigned a score that indicated a survival prognosis. The estimated individual probability of survival at each time point could be determined by summing up the points assigned to each variable.

Comparison of the predictive ability of our nomogram with that of other prognostic models for post-LT survival in patients satisfying the APASL criteria of $H B V-A C L F$

The determiner calibration curve showed an optimal agreement between prediction and actual observation in the probability of 28-day, 3-month, 6-month and 1-year survival in nomogram (Figure 3). Compared with 5 other prognostic models, the established nomogram displayed better predictive accuracy in predicting post-LT survival. The C-index of the nomogram (0.707) for predicting 1 -year survival was significantly higher than those of the CPS (0.626, $\mathrm{P}=0.004)$, MELD score $(0.627, \mathrm{P}<0.001)$, MELD-Na $(0.583, \mathrm{P}<0.001)$, and CLIF-C OF $(0.674$, $\mathrm{P}=0.034)$. There was no significant difference regarding $\mathrm{C}$-indices between the nomogram and CLIF-C ACLF (0.684, $\mathrm{P}=0.064)$. The $\mathrm{C}$-indices of the nomogram also showed similar results in the prediction of 28-day (0.73), 3 -month (0.722), and 6-month (0.708) survival compared with other models (Table 3). These results demonstrated that the nomogram had an advantage over other models as a predictor of post-LT survival in patients with HBV-ACLF.

\section{Validation of the nomogram in patients satisfying the EASL criteria of $H B V-A C L F$}

Since the majority (75.2\%) of the patients satisfied the EASL criteria for ACLF, these patients were used to investigate the prognostic discrimination ability of the nomogram. The C-index of the nomogram (0.692) was significantly higher than those of CPS $(0.617,95 \%$ CI: $0.680-0.739, \mathrm{P}=0.030)$, MELD $(0.598,95 \% \mathrm{CI}$ : $0.589-0.607, \mathrm{P}=0.002)$, and MELD-Na $(0.566,95 \% \mathrm{CI}$ : $0.557-0.575, \mathrm{P}=0.006)$ in predicting post-LT 1 -year survival (Table 3). The C-index of the nomogram was also superior to those of CLIF-C OF $(0.658, \mathrm{P}=0.072)$ and CLIF-C ACLF (0.674, $\mathrm{P}=0.142$ ), albeit not significantly. Similar results were observed in the prediction of 28 -day, 3-month, and 6-month survival. These results suggested that the nomogram was useful for predicting post-LT survival in patients who satisfied the EASL criteria for HBV-ACLF.

\section{Predictive performance of the nomogram in stratifying risk among patients}

In order to analyze the discriminative ability of the nomogram, participants were grouped into 3 different subgroups according to cutoff values of total points (Figure S2). The Kaplan-Meier curves for each group were plotted, representing a discrepant prognosis (Figure 4). The nomogram accurately stratified patients into 3 distinct prognostic subgroups (1-year post-LT survival rate of $64.86 \%, 24.81 \%$, and $8.87 \%$, respectively; log-rank $\mathrm{P}<0.001)$. 
Table 2 Univariate and multivariate analysis of variables to predict the 1-year post-transplant survival of HBV-ACLF patients

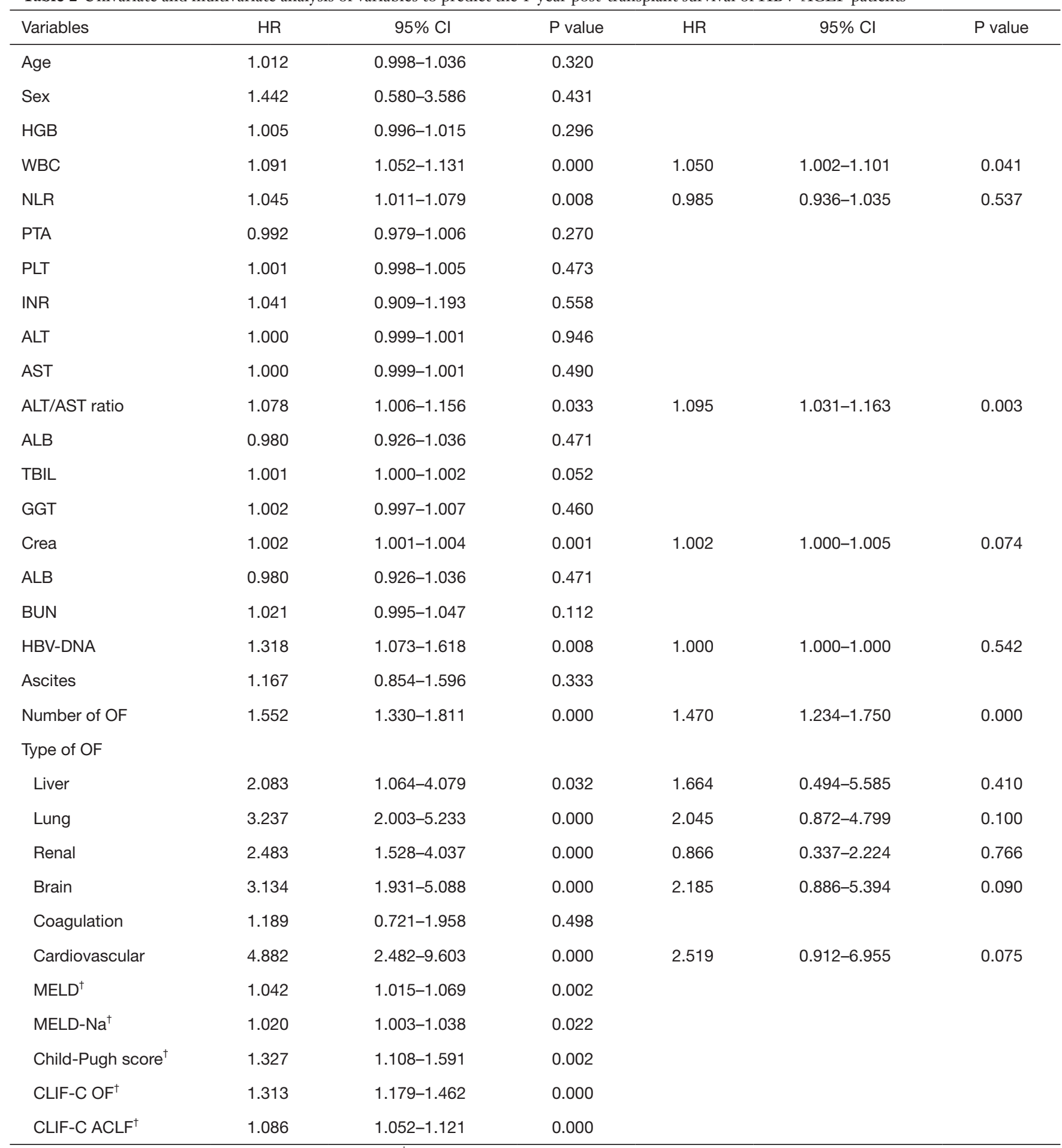

$\mathrm{P}$ values $<0.05$ represent statistical significance. ${ }^{\dagger}$, the algorithms calculated on baseline serum parameters were not included in the multivariable Cox proportional hazard regression analysis. ACLF, acute-on-chronic liver failure; ALB, albumin; ALT, alanine transaminase; AST, aspartate transaminase; BUN, blood urea nitrogen; $\mathrm{Cl}$, confidence interval; CLIF-C OF, chronic liver failure Consortium Organ Failure score; EASL, European Association for the Study of the Liver; GGT, gamma-glutamyl transpeptidase; HBV, hepatitis B virus; HGB, hemoglobin; HR, hazard ratio; INR, international normalized ratio; MELD, model for end stage liver disease; NLR, neutrophil-to-lymphocyte ratio; OF, organ failure; PTA, prothrombin activity; PLT, platelet; TBIL, total bilirubin; WBC, white blood cell. 


Points
WBC
ALT/AST ratio
Organ failure numbers

Figure 2 Prognostic nomogram for 28-day, 90-day, 180-day, and 1-year post-transplant survival in HBV-ACLF patients. To use the nomogram, locate an individual patient's value on each variable axis, and draw a line upward to determine the points associated with each variable value. Locate the sum of the points together on the total points axis. Draw a line straight down to determine the likelihood of 28 day, 90-day, 180-day, and 1-year post-transplant survival. ALT/AST ratio, alanine aminotransferase to aspartate aminotransferase ratio; OF, organ failure; OS, overall survival; WBC, white blood cells.

\section{Development of an online calculator for our nomogram}

To support its application in clinical practice, we developed an online version of our nomogram. Clinicians can access the nomogram (at https://hbv-aclf-nomogram.shinyapps. io/dynnomapp/) and input clinical features to obtain instant prognostic survival curves generated by the webserver.

\section{Discussion}

To the best of our knowledge, this is the first large sample study to describe the development and validation of a prognostic nomogram for predicting post-LT survival in patients with HBV-ACLF. Its C-index was greater than those of the conventional prognostic models and was comparable to that of CLIF-C ACLF. The nomogram could be implemented to assist physicians to carry out individualized survival prediction and identify patients who are potentially unsuitable for LT.

The predictive accuracy of the nomogram for postLT survival can be explained by the factors included in the model. A high level of WBC is a simple and evident indicator of systemic inflammation (16) that is constantly neglected by traditional prognostic scores. In accordance with previous studies $(2,18,19)$, our study found that higher a WBC count was associated with more severe complications and a higher ACLF grade. With more extrahepatic organ involvement and oxidative stress, patients with HBV-ACLF are more prone to developing systemic inflammatory response syndrome (SIRS) than those with hepatitis due to other causes (20). An increased WBC count and excessive production of proinflammatory cytokines then induce tissue damage in both the liver and remote organs $(21,22)$, leading to a higher rate of organ failure and mortality. Thus, incorporation of WBC count into the nomogram added prognostic weight.

Interestingly, our findings revealed that the preoperative ALT/AST ratio was associated with the outcomes of patients with HBV-ACLF who underwent LT. The ALT/ AST ratio is not only a marker of liver fibrosis and cirrhosis, but it also provides prognostic information $(23,24)$. Our 
A

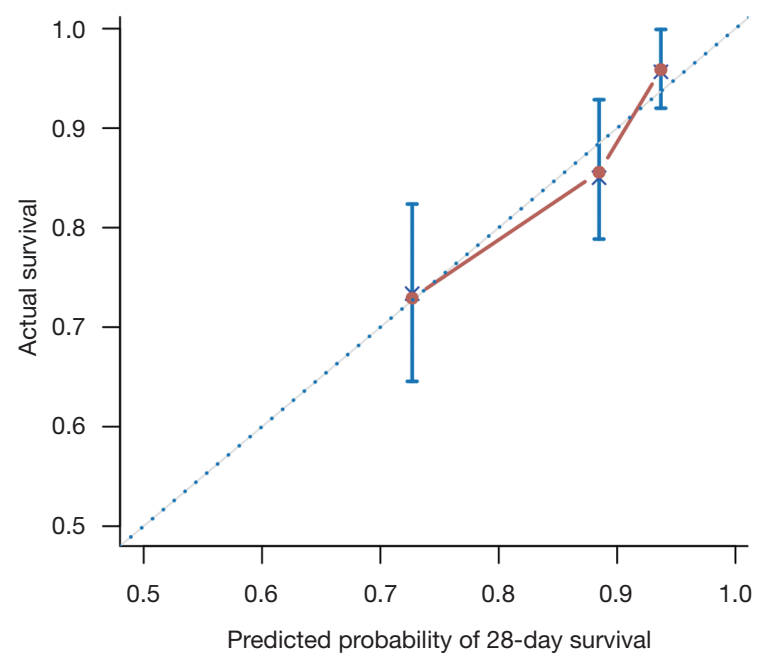

C

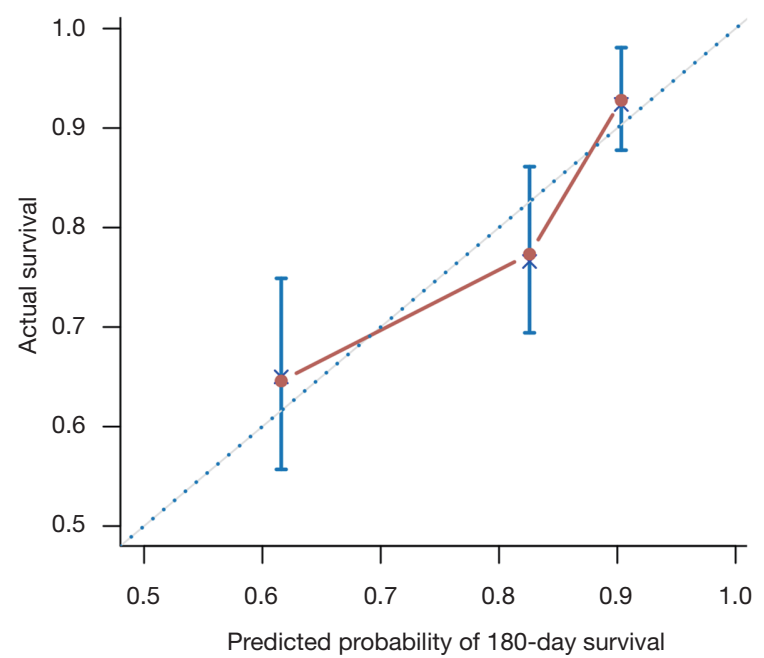

B

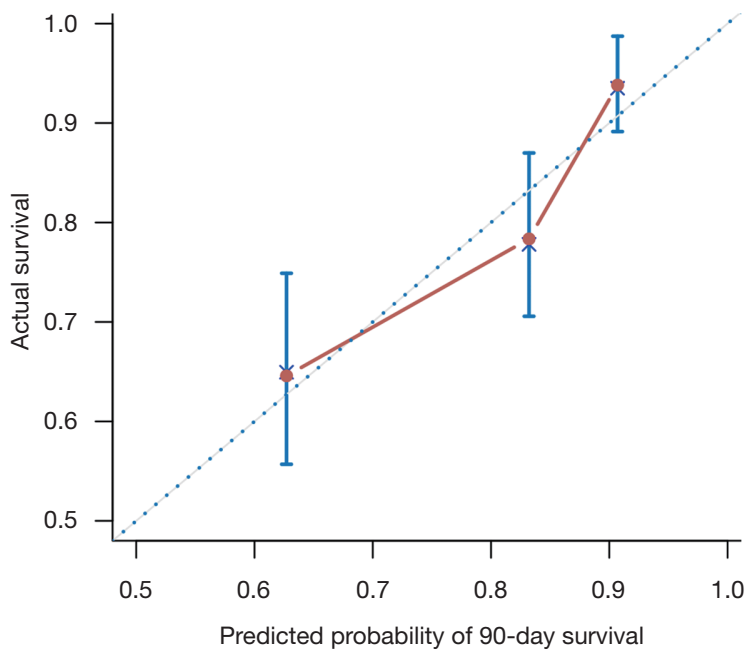

D

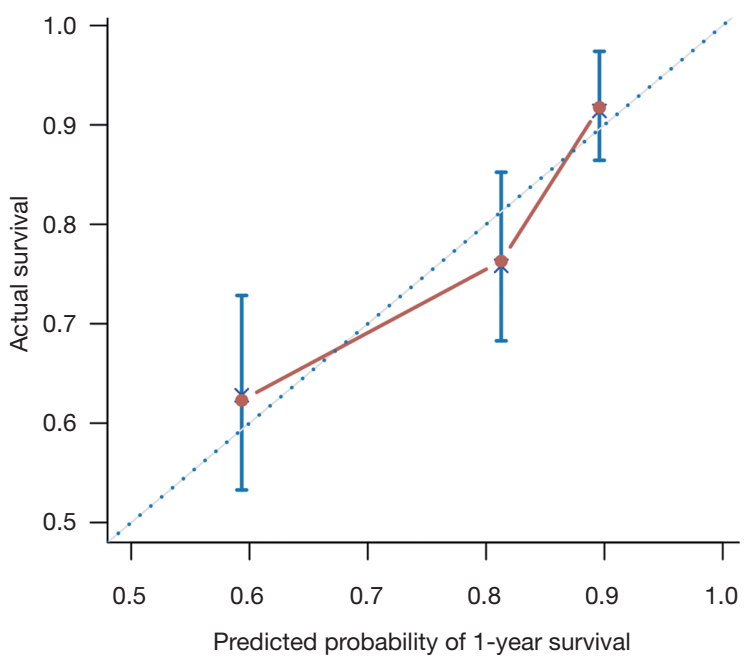

Figure 3 Determiner calibration curves of the nomogram for predicting 28-day, 90-day, 180-day, and 1-year post-transplant survival in HBV-ACLF patients. The average predicted probability of OS was plotted on the $\mathrm{x}$-axis, and the observed OS was plotted on the $\mathrm{y}$-axis. ACLF, acute-on-chronic liver failure; HBV, hepatitis B virus; OS, overall survival.

study found that the ALT/AST ratio was a significant risk factor for prognosis in univariate and multivariate analyses. A previous study suggested that a high ALT/AST ratio may reflect the time course of acute viral hepatitis and indicate a poor prognosis (25). The elevated ALT/AST ratio could be partly explained by increased mitochondrial release of AST during the acute phase of ACLF. However, whether the predictive value of the ALT/AST ratio depends on etiology requires more validation in independent datasets.
In spite of improvements in medical management, patients with ACLF frequently develop multi-organ failure and have poor short-term outcomes. To date, an effective method of predicting post-LT survival in patients with HBV-ACLF has not been described. The CLIF-C ACLF was derived from Western populations in which alcoholic ACLF and HCV-related ACLF are predominant; thus, it represents an accurate prognostic tool for ACLF patients. This study showed that preoperative CLIF-C ACLF 


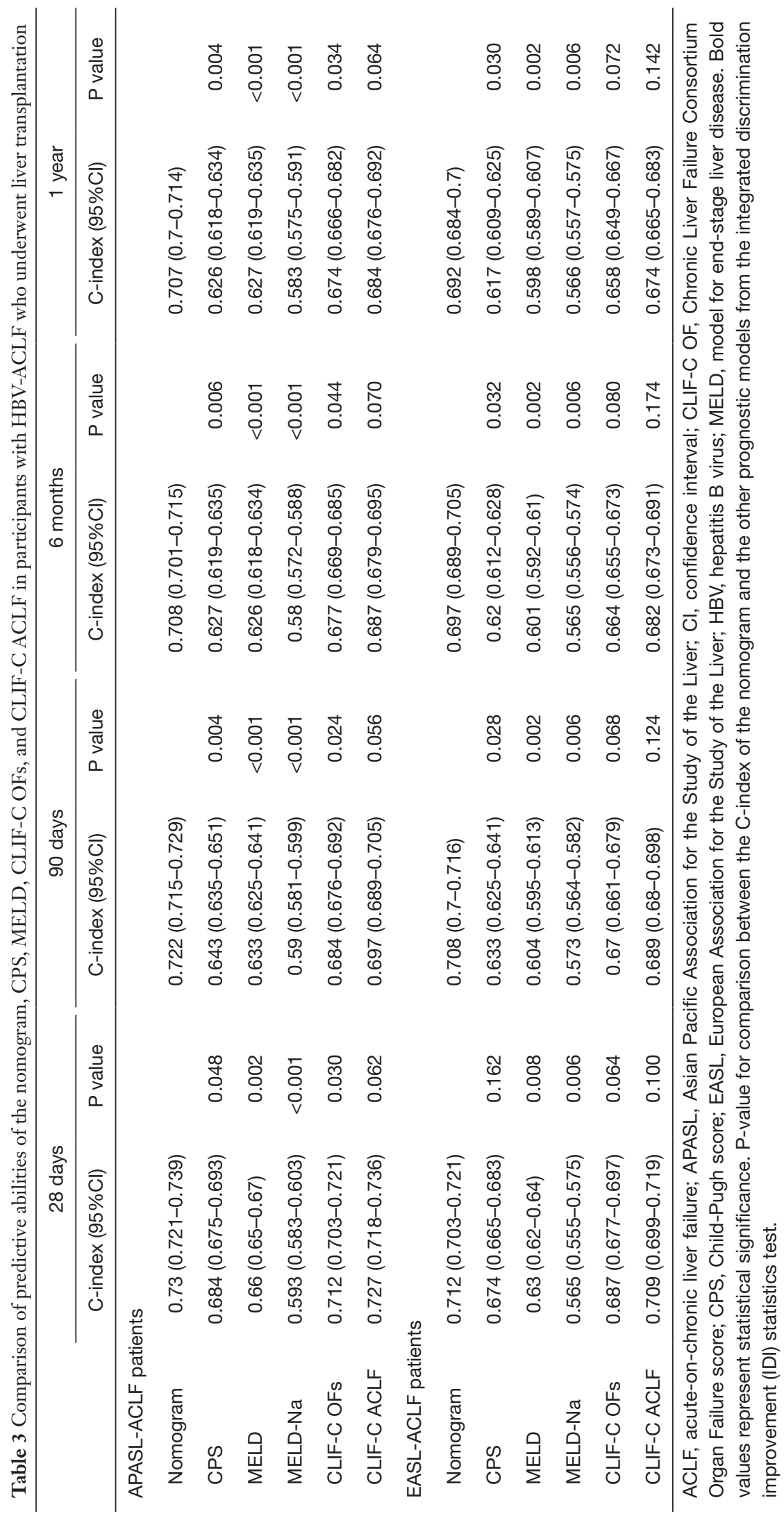




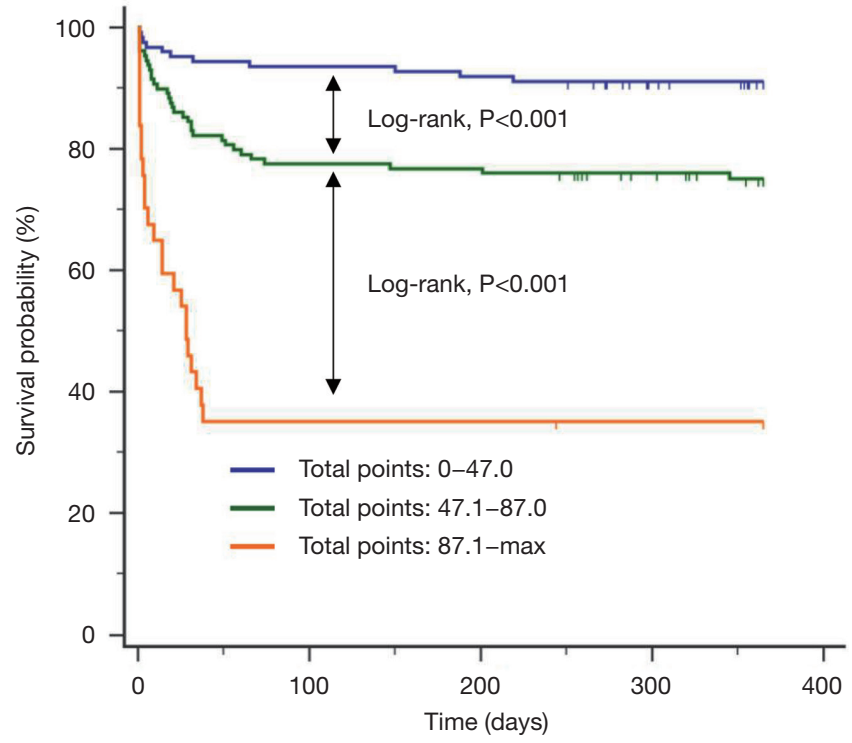

Figure 4 Kaplan-Meier survival curves of patients stratified by the cut-off values of total points. The curves showed different survival rates of patients with total points trisection cut-off values (high risk:0-47.0; intermediate risk: 47.1-87.0; and low risk: $\geq 87.1$ ).

also had good predictive accuracy for post-LT survival in patients with HBV-ACLF, which was similar to that of the nomogram. Consistent with previous studies $(1,26)$, our results indicated that OF was significantly associated with the prognosis of patients with ACLF (Table S2). The organ failure numbers were found to make the greatest contribution to prognostic prediction in the nomogram, in which 15 points were gained for each organ with failure. In the subgroup of patients with failure of 4 organs, 1-year mortality did not increase accordingly, which could be explained by the inadequate sample size of this study.

It is noteworthy that for patients with failure of more than 3 organs, the CPS, MELD, and MELD-Na scores showed no significant differences, meaning these patients may not be given priority based on the current organ allocation system, even though they have a higher risk of mortality. Since patients with failure of 3 or more organs account for $18.4 \%$ of those who are listed for LT in the United Network for Organ Sharing (UNOS) (27), additional research is warranted to address the limitations of the system. Whether the MELD-based allocation policy should be aimed at the "sickest first" or at the "maximization of utility" remains controversial (10). Recent studies have indicated that under careful selection, patients with multiple organ failure or ACLF grade 3 could benefit from LT
$(26,27)$. However, the criteria for listing and ideal timing of LT for HBV-ACLF patients with multiple organ failure has not been fully elucidated (28). It would not only be medically but also ethically challenging for doctors to consider the option of LT for these patients. Meanwhile, the inclusion of the organ failure numbers in the nomogram implies that organ support and intensive monitoring are crucial for ACLF patients on the waiting list, because therapeutic strategies for bridging the patient to LT could potentially improve survival.

An increasing number of studies have suggested that the type of organ failure is also an important factor that may influence patient survival after transplantation (19). The need for mechanical ventilation, which is an indicator of respiratory failure, was found to be associated with reduced post-transplant survival $(27,29)$. However, whether respiratory failure should be recognized as a contraindication for LT remains controversial and requires further elucidation. We found that patients with cardiovascular, brain, and lung failure had higher 1-year post-LT mortality than those with other types of organ failure, although these factors were not included in the nomogram. These results conform to previous findings and highlight the need for careful decision-making regarding transplantation for patients presenting with certain types of organ failure (30). However, analysis by Thuluvath et al. demonstrated that type of organ failure may have less bearing in determining post-transplant survival, which can be partly explained by discrepancy in the definition of respiratory failure (27).

Sundaram et al. found that patients who recovered from ACLF grade 3 at registration to ACLF $0-2$ at transplantation had a significantly higher 1-year survival after transplantation than those who did not (30). Despite alterations in ACLF grade from registration to transplantation, the final ACLF grade at LT had a more decisive role in predicting survival. A longer duration of the final ACLF grade developed by the patient before transplantation may indicate poorer post-LT survival, although the relevant data did not show statistical significance. Our nomogram was derived from clinical features recorded within the 1 week before transplantation and did not include data from the time of waiting list registration. The predictive ability of the nomogram is more reflective of the final ACLF grade before LT than ACLF grade at registration. If patients with ACLF grade 3 at registration downgrade to ACLF grades 0-2 before LT, the nomogram score will be lower in line with the decreased 
organ failure numbers. These downgraded patients will have a better survival probability based on our nomogram.

Nomograms are regularly used pictorial tools to estimate prognosis and have been shown to be more accurate than traditional models in predicting individualized risk in ACLF and acute decompensation $(13,31)$. This study constructed and internally validated a novel nomogram including WBC count, ALT/AST ratio, and the organ failure numbers. Our nomogram showed a better ability in predicting 3-month, 6-month, and 1-year mortality than CPS, MELD, MELD$\mathrm{Na}$, and CLIF-C OFs, with good discrimination and determiner calibration. With comparable predictive ability, the nomogram is easier-to-use than CLIF-C ACLF for the calculation of mortality risk, as it is presented as a visualized scale. The Technology Acceptance Model (TAM) score, which consists of arterial lactate level, mechanical ventilation, age, and leukocyte counts, was reported to have better predictive ability than CLIF-C ACLF in ACLF grade 3 patients' post-LT outcomes (32). Due to the limitation of data availability, we were unable to compare this model with our nomogram in the HBV-ACLF patient cohort.

This study had several limitations. First, the patient cohort was derived from a single center. Thus, the nomogram is likely to have been influenced by clinical management and perioperative care made by that single center, and it requires multi-center validation in the future studies. Second, since ACLF is a highly dynamic disease process, data in this study were collected from a single preoperative time point instead of sequential time points because preoperative treatment may also lead to variation of related parameters. In addition, some important molecular factors (such as serum cytokine concentration) were lacking due to the retrospective nature of the study. Also, since the nomogram was derived from HBV-ACLF patients, it may not be applicable for ACLF patients with other etiologies such as hepatitis $\mathrm{C}$ virus (HCV) or alcoholic liver disease. Despite these limitations, our study had a large sample size with detailed information about patient characteristics and OF at the time of LT.

\section{Conclusions}

In conclusion, our study proposed a novel nomogram that could accurately predict individualized post-LT survival in patients with HBV-ACLF. The nomogram might be a useful tool for identifying HBV-ACLF patients who would benefit from LT. Additional studies are required to validate and refine the nomogram in other multi-center cohorts.

\section{Acknowledgments}

Funding: This work was supported by the grants from the National Key R\&D Program of China (2017YFA0104304), National Natural Science Foundation of China (81770648, 81802402, 81870449, 81901943, 81900597, 81970567), National Key R\&D Program of Guangdong Province (2019B020236003), Guangdong Natural Science Foundation (2017A030311034, 2018A03031032, 2018A030313043), Medical Scientific Research Foundation of Guangdong Province (A2018130), Sci-tech Research Development Program of Guangzhou City (158100076), and Academician Shusen Lanjuan Talent Foundation.

\section{Footnote}

Reporting Checklist: The authors have completed the Transparent Reporting of a multivariable prediction model for Individual Prognosis or Diagnosis (TRIPOD) Checklist. Available at http://dx.doi.org/10.21037/atm-20-6180

Data Sharing Statement: Available at http://dx.doi. org/10.21037/atm-20-6180

Peer Review File: Available at http://dx.doi.org/10.21037/ atm-20-6180

Conflicts of Interest: All authors have completed the ICMJE uniform disclosure form (available at http://dx.doi. org/10.21037/atm-20-6180). All authors report a pending patent "The Nomogram in Survival Prediction for Posttransplant HBV-ACLF Patients".

Ethical Statement: The authors are accountable for all aspects of the work in ensuring that questions related to the accuracy or integrity of any part of the work are appropriately investigated and resolved. The study was conducted in accordance with the Declaration of Helsinki (as revised in 2013). The study was approved by institutional ethics committee of The Third Affiliated Hospital of Sun Yat-Sen University (No. 2018-353-02), and individual consent for this retrospective analysis was waived. The study outcomes will not affect the future management of the patients. All patient data were obtained from the medical record system of The Third Affiliated Hospital of Sun YatSen University and have been secured.

Open Access Statement: This is an Open Access article distributed in accordance with the Creative Commons 
Attribution-NonCommercial-NoDerivs 4.0 International License (CC BY-NC-ND 4.0), which permits the noncommercial replication and distribution of the article with the strict proviso that no changes or edits are made and the original work is properly cited (including links to both the formal publication through the relevant DOI and the license). See: https://creativecommons.org/licenses/by-nc-nd/4.0/.

\section{References}

1. Wu T, Li J, Shao L, et al. Development of diagnostic criteria and a prognostic score for hepatitis B virus-related acute-on-chronic liver failure. Gut 2018;67:2181-91.

2. Moreau R, Jalan R, Gines P, et al. Acute-on-chronic liver failure is a distinct syndrome that develops in patients with acute decompensation of cirrhosis. Gastroenterology 2013;144:1426-37, 37.e1-9.

3. Arroyo V, Moreau R, Jalan R, et al. Acute-on-chronic liver failure: A new syndrome that will re-classify cirrhosis. J Hepatol 2015;62:S131-43.

4. Gustot T, Fernandez J, Garcia E, et al. Clinical Course of acute-on-chronic liver failure syndrome and effects on prognosis. Hepatology 2015;62:243-52.

5. Finkenstedt A, Nachbaur K, Zoller H, et al. Acuteon-chronic liver failure: excellent outcomes after liver transplantation but high mortality on the wait list. Liver Transpl 2013;19:879-86.

6. Petrowsky H, Rana A, Kaldas FM, et al. Liver transplantation in highest acuity recipients: identifying factors to avoid futility. Ann Surg 2014;259:1186-94.

7. McPhail MJ, Auzinger G, Bernal W, et al. Decisions on futility in patients with cirrhosis and organ failure. Hepatology 2016;64:986.

8. Rana A, Jie T, Porubsky M, et al. The survival outcomes following liver transplantation (SOFT) score: validation with contemporaneous data and stratification of high-risk cohorts. Clin Transplant 2013;27:627-32.

9. Halldorson JB, Bakthavatsalam R, Fix O, et al. D-MELD, a simple predictor of post liver transplant mortality for optimization of donor/recipient matching. Am J Transplant 2009;9:318-26.

10. Linecker M, Krones T, Berg T, et al. Potentially inappropriate liver transplantation in the era of the "sickest first" policy - A search for the upper limits. J Hepatol 2018;68:798-813.

11. Balachandran VP, Gonen M, Smith JJ, et al. Nomograms in oncology: more than meets the eye. The Lancet Oncology 2015;16:e173-80.
12. van Vugt JLA, Alferink LJM, Buettner S, et al. A model including sarcopenia surpasses the MELD score in predicting waiting list mortality in cirrhotic liver transplant candidates: a competing risk analysis in a national cohort. J Hepatol 2018;68:707-14.

13. Cai YJ, Dong JJ, Dong JZ, et al. A nomogram for predicting prognostic value of inflammatory response biomarkers in decompensated cirrhotic patients without acute-on-chronic liver failure. Aliment Pharmacol Ther 2017;45:1413-26.

14. Sarin SK, Kedarisetty CK, Abbas Z, et al. Acute-onchronic liver failure: consensus recommendations of the Asian Pacific Association for the Study of the Liver (APASL) 2014. Hepatol Int 2014;8:453-71.

15. Carlson RV, Boyd KM, Webb DJ. The revision of the Declaration of Helsinki: past, present and future. Br J Clin Pharmacol 2004;57:695-713.

16. Jalan R, Saliba F, Pavesi M, et al. Development and validation of a prognostic score to predict mortality in patients with acute-on-chronic liver failure. J Hepatol 2014;61:1038-47.

17. Uno H, Tian L, Cai T, et al. A unified inference procedure for a class of measures to assess improvement in risk prediction systems with survival data. Stat Med 2013;32:2430-42.

18. Cordoba J, Ventura-Cots M, Simon-Talero M, et al. Characteristics, risk factors, and mortality of cirrhotic patients hospitalized for hepatic encephalopathy with and without acute-on-chronic liver failure (ACLF). J Hepatol 2014;60:275-81.

19. Shi $Y$, Yang $Y, H u ~ Y$, et al. Acute-on-chronic liver failure precipitated by hepatic injury is distinct from that precipitated by extrahepatic insults. Hepatology 2015;62:232-42.

20. Choudhury A, Kumar M, Sharma BC, et al. Systemic inflammatory response syndrome in acute-on-chronic liver failure: Relevance of 'golden window': A prospective study. J Gastroenterol Hepatol 2017;32:1989-97.

21. Clària J, Arroyo V, Moreau R. The Acute-on-Chronic Liver Failure Syndrome, or When the Innate Immune System Goes Astray. J Immunol 2016;197:3755-61.

22. Sarin SK, Choudhury A. Acute-on-chronic liver failure: terminology, mechanisms and management. Nat Rev Gastroenterol Hepatol 2016;13:131-49.

23. Angulo P, Keach JC, Batts KP, et al. Independent predictors of liver fibrosis in patients with nonalcoholic steatohepatitis. Hepatology 1999;30:1356-62 .

24. Angulo P, Bugianesi E, Bjornsson ES, et al. Simple 
noninvasive systems predict long-term outcomes of patients with nonalcoholic fatty liver disease. Gastroenterology 2013;145:782-9.e4.

25. Botros M, Sikaris KA. The de ritis ratio: the test of time. Clin Biochem Rev 2013;34:117-30.

26. Artru F, Louvet A, Ruiz I, et al. Liver transplantation in the most severely ill cirrhotic patients: A multicenter study in acute-on-chronic liver failure grade 3. J Hepatol 2017;67:708-15.

27. Thuluvath PJ, Thuluvath AJ, Hanish S, et al. Liver transplantation in patients with multiple organ failures: Feasibility and outcomes. J Hepatol 2018;69:1047-56.

28. Gustot T, Moreau R. Acute-on-chronic liver failure vs. traditional acute decompensation of cirrhosis. J Hepatol 2018;69:1384-93.

Cite this article as: Chen L, Zhang J, Lu T, Cai J, Zheng J, Yao J, Yi S, Li H, Chen G, Zhao H, Zhang Y, Yang Y. A nomogram to predict survival in patients with acuteon-chronic hepatitis B liver failure after liver transplantation. Ann Transl Med 2021;9(7):555. doi: 10.21037/atm-20-6180
29. Sundaram V, Jalan R, Wu T, et al. Factors Associated with Survival of Patients With Severe Acute-On-Chronic Liver Failure Before and After Liver Transplantation. Gastroenterology 2019;156:1381-91.e3.

30. Sundaram V, Kogachi S, Wong RJ, et al. Effect of the clinical course of acute-on-chronic liver failure prior to liver transplantation on post-transplant survival. J Hepatol 2020;72:481-8.

31. Shi KQ, Cai YJ, Lin Z, et al. Development and validation of a prognostic nomogram for acute-on-chronic hepatitis B liver failure. J Gastroenterol Hepatol 2017;32:497-505.

32. Artzner T, Michard B, Weiss E, et al. Liver transplantation for critically ill cirrhotic patients: Stratifying utility based on pretransplant factors. Am J Transplant 2020;20:2437-48. 


\section{Supplementary}

Table S1 Number and mortality of patients with different ACLF grades under EASL criteria

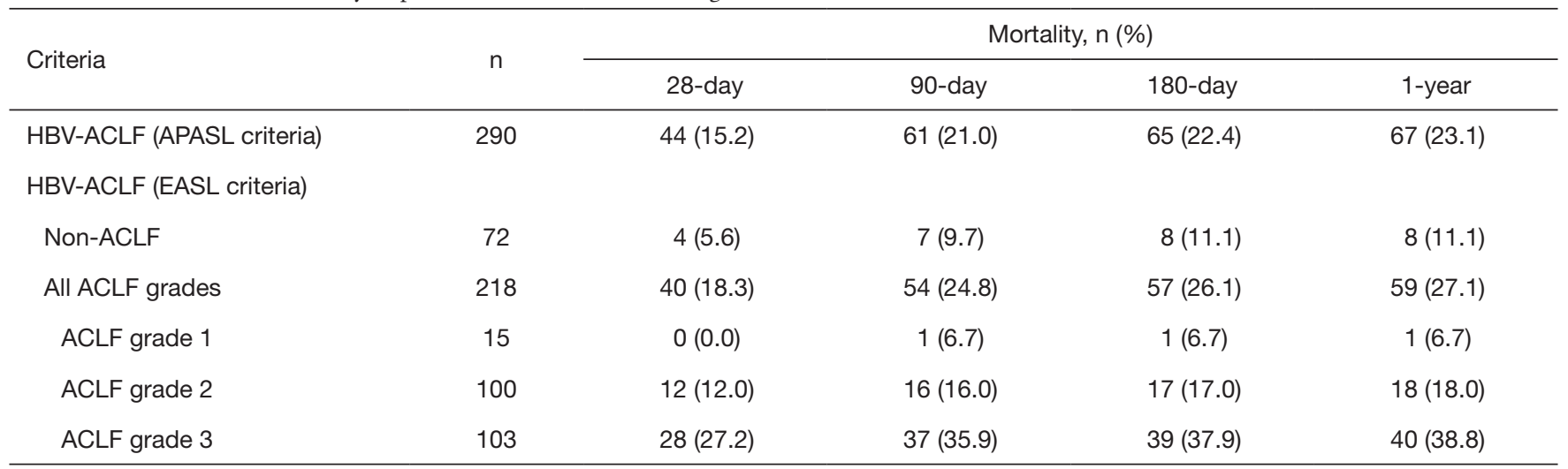

ACLF, acute-on-chronic liver failure; APASL, Asian Pacific Association for the Study of the Liver; EASL, European Association for the Study of the Liver; HBV, hepatitis B virus.

Table S2 One-year post-transplant mortality, MELD, MELD-Na, Child-Pugh score of HBV-ACLF patients with different number of organ failures

\begin{tabular}{lccccc}
\hline Number of organ failure & $\mathrm{n}$ & 1-year mortality & MELD & MELD-Na & Child-Pugh score \\
\hline 0 & 44 & $13.64 \%$ & $16.5(13.3-23.0)^{\star}$ & $12.4(5.3-22.6)^{\star}$ & $10.0(9.0-11.0)$ \\
1 & 43 & $6.98 \%$ & $29.0(24.0-33.0)^{\star}$ & $30.1(21.7-26.2)$ & $11.0(10.0-12.0)$ \\
2 & 100 & $18.00 \%$ & $35.0(32.0-39.5)^{\star}$ & $34.0(26.2-42.3)$ & $11.0(10.2-12.0)$ \\
3 & 56 & $32.14 \%$ & $39.5(34.0-44.8)$ & $36.4(25.5-45.1)$ & $12.0(11.0-13.0)$ \\
4 & 19 & $21.05 \%$ & $42.0(38.0-49.0)$ & $37.9(32.0-44.6)$ & $12.0(12.0-13.0)$ \\
5,6 & 28 & $62.29 \%$ & $44.5(39.3-49.8)$ & $40.6(34.1-46.0)$ & $13.0(12.0-13.0)$ \\
\hline
\end{tabular}

*, $\mathrm{P}$ value $(<0.05)$ for comparisons between patients with one more organ failure. Where applicable, data are presented as median (interquartile range). ACLF, acute-on-chronic liver failure; HBV, hepatitis B virus; MELD, model for end-stage liver disease. 


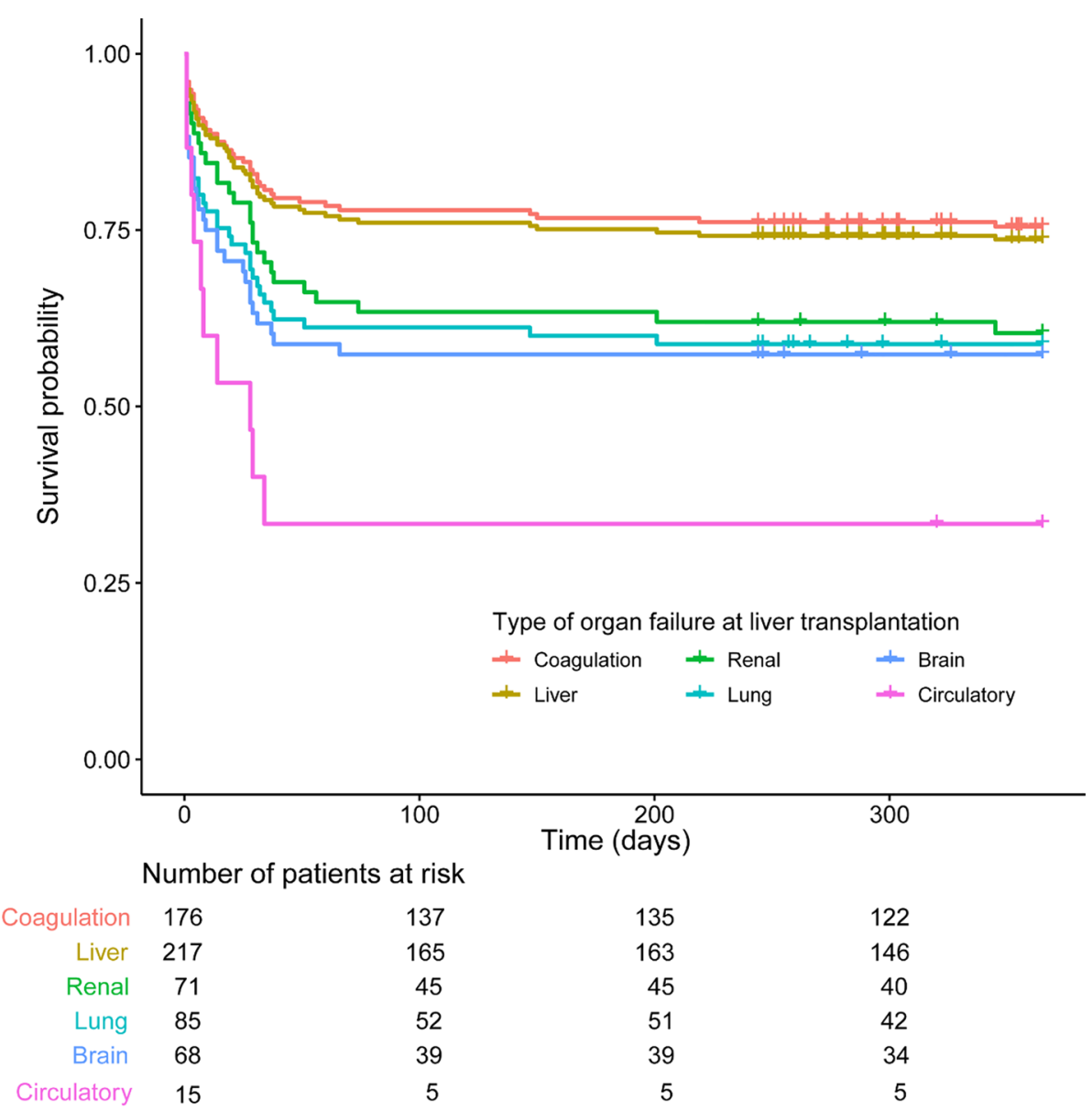

Figure S1 Patient survival probability stratified by the type of organ failure at the time of liver transplantation.

Table S3 Twenty-eight-day, 90-day, 6-month, and 1-year post-transplant mortality of HBV-ACLF patients with different type of organ failure

\begin{tabular}{|c|c|c|c|c|c|}
\hline Type of organ failure & $\mathrm{n}$ & 28-day mortality & 90-day mortality & 6-month mortality & 1-year mortality \\
\hline Liver & 217 & $17.97 \%$ & $23.96 \%$ & $25.35 \%$ & $26.27 \%$ \\
\hline Lung & 85 & $30.59 \%$ & $38.82 \%$ & $41.18 \%$ & $41.18 \%$ \\
\hline Renal & 71 & $23.94 \%$ & $36.62 \%$ & $38.03 \%$ & $39.44 \%$ \\
\hline Brain & 68 & $35.29 \%$ & $42.65 \%$ & $42.65 \%$ & $42.65 \%$ \\
\hline Cardiovascular & 15 & $53.33 \%$ & $66.67 \%$ & $66.67 \%$ & $66.67 \%$ \\
\hline
\end{tabular}

ACLF, acute-on-chronic liver failure; HBV, hepatitis B virus. 
A

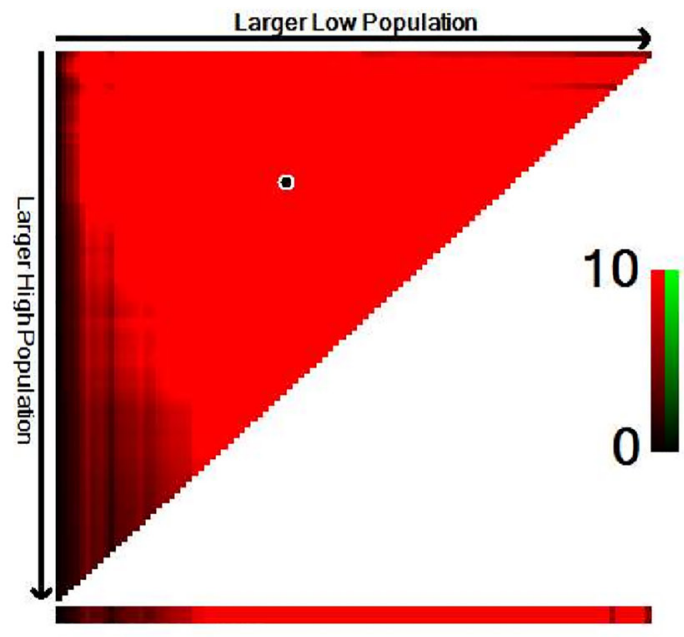

B

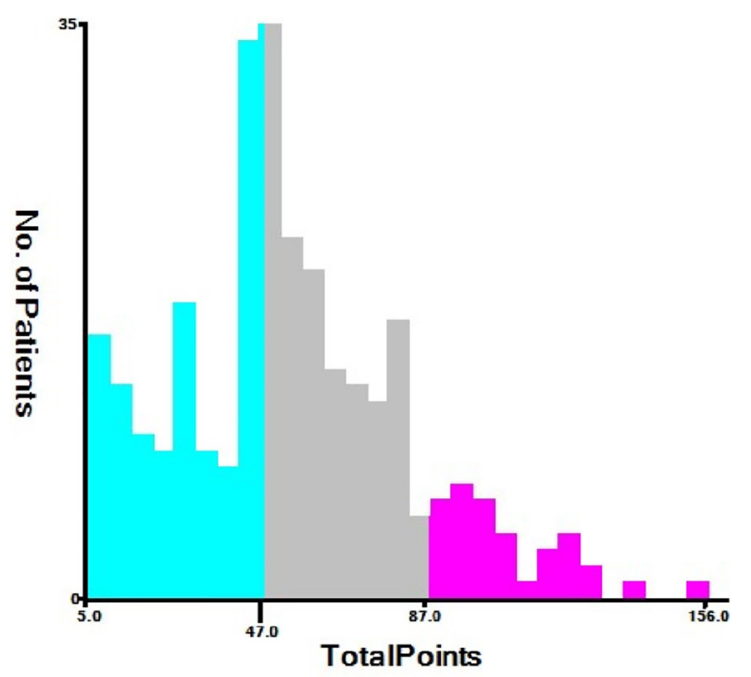

Figure S2 Cut-off value of total points calculated using the X-tile software. Coloration of the plot (A) represents the strength of the association at each division, ranging from low (dark, black) to high (green). The trisection cut-off value (blue: 0-47.0; grey: 47.1-87.0; red: $\geq 87.1$ ) was demonstrated on a histogram (B) of all recruited patients 